\title{
Publication Name
}

National Cancer Institute

\section{Source}

National Cancer Institute. Publication Name. NCI Thesaurus. Code C93639.

A human readable textual identifier of the publication. 